\title{
CUANTIFICACIÓN DE SUSTANCIAS MARCADORAS DE ENVEJECIMIENTO EN Quercus humboldtii POR CROMATOGRAFÍA LÍQUIDA DE ALTA EFICIENCIA
}

\section{QUANTIFICATION OF AGING MARKERS SUBSTANCES IN Quercus humboldtii THROUGH HIGH EFFICIENCY LIQUID CHROMATOGRAPHY}

\author{
Rafael E. González', Lilia S. Calderón, Rodolfo A. Cabeza. \\ Recibido para evaluación: Septiembre 16 de 2008 - Aceptado para publicación: Diciembre 16 de 2008
}

\begin{abstract}
RESUMEN
En la etapa de maduración de las bebidas alcohólicas se utilizan barriles de roble Americanos y Franceses, que al contacto con el aguardiente liberan sustancias que finalmente contribuyen con las características sensoriales del mismo. El siringaldehido y la vainillina son algunos de estos compuestos que a su vez son considerados como marcadores de envejecimiento ya que son útiles para evaluar la calidad de las bebidas envejecidas. El objetivo de este trabajo fue investigar por primera vez en Colombia la presencia de estos compuestos en el roble Colombiano (Quercus humboldtii) tostado a $180^{\circ} \mathrm{C}$ por tres horas y $\sin$ tostar, por Cromatografía Líquida de Alta Eficiencia. Iniciando un proceso de evaluación sobre la posibilidad de utilizar el roble colombiano en la elaboración de barriles para maduración de bebidas alcohólicas. Encontrándose que el tostado influencia decisivamente la composición de Quercus humboldtii, debido a que no se detectaron concentraciones de siringaldehido y vainillina en roble sin tostar. Además se presentó una relación siringaldehido/vainillina de 1.45 en el roble tostado, que demuestra un equilibrio entre estos aldehídos y la posibilidad de continuar estudios sobre la aceptación de este material.
\end{abstract}

Palabras clave: siringaldehído, tostado, vainillina.

\begin{abstract}
During maturation of alcoholic drinks, American and French oak barrels are used. These in contact with the liquor release substances that contribute with final drink tasting characteristics. Syringaldehyde and vainillin are some of these compounds that are considered aging markers to determine quality of aged
\end{abstract}

${ }^{1}$ Universidad de Pamplona, Facultad de Ciencias Básicas, Km 1 vía Bucaramanga, Pamplona - Norte de Santander, Tel. ( ) 5585303 E-mail: rgonzales@unipamplona.edu.co. 
drinks. The objective of this work was to determine, using high performance liquid chromatography, the presence of both, syringaldehyde and vainillin, compounds in Colombian oak (Quercus humboldtii) with and without toasting at $180^{\circ} \mathrm{C}$ during 3 hours, in order to evaluate the possibility of using Colombian oak barrels for drink maturation. The data showed that toasting significantly influences characteristics of Colombian oak, since no syringaldehyde and vainillin were detected when toasting was not applied. Syringaldehyde/ vanillin relation was 1.45 in toasted indicating a balance between these aldehydes and the possibility of continuing studies regarding acceptance of Querqus humboldtii as source for drink maturation barrels.

Keys words: syringaldehyde, toasting, vainillin.

\section{INTRODUCCIÓN}

Aunque históricamente se encuentra documentado el uso de especies madereras muy distintas con destino a la construcción de barricas para la maduración de bebidas alcohólicas, actualmente se utiliza mayoritariamente la madera de roble, debido a los efectos positivos que tiene sobre la bebida final (Garde y Ancín, 2006). Esto se debe principalmente a la migración de compuestos volátiles que pasan del roble a la bebida alcohólica, jugando un papel importante en sus características organolépticas y/o sensoriales. Particularmente la cantidad de estos compuestos dependen de las especies de roble, el origen geográfico, el tostado y secado de la madera durante el proceso de fabricación de los barriles (natural o artificial) y el tipo de madera (especie y origen) (Chatonnet et al., 1994; Mosedale y Savill, 1996; Chatonnet y Dubourdieu, 1998; Pérez et al., 1999; Cadahia et al., 2001; Fernández de Simón et al., 2003; Ancín et al., 2004).

El siringaldehído y la vainillina han sido considerados tradicionalmente como sustancias marcadoras de envejecimiento en bebidas alcohólicas destiladas envejecidas, y son utilizados como un buen parámetro para señalar la calidad y autenticidad de las bebidas; ya que su cuantificación durante el proceso de envejecimiento puede ser usada para evaluar el tiempo requerido de envejecimiento de una bebida destilada, es decir cuando sus concentraciones aumentan con el tiempo de envejecimiento. Además la relación entre las concentraciones de estos dos compuestos ha sido utilizada como un indicador de la calidad de las bebidas espirituosas y envejecimiento genuino en madera (Bozhinov y Belakov, 1983; Delgado et al., 1990; Lo coco et al., 1995; Quesada et al., 1996; Mangas et al., 1996; Jaganathan y Dugar, 1999; Batista de Aquino et al., 2006).

Los robles más utilizados para la crianza de vinos y aguardientes son el roble Francés (Quercus sessilis y Quercus robur) y el Americano (Quercus alba). Actualmente las dos áreas más importantes que suministran madera, destinada a la fabricación de barriles son Europa y Estados Unidos. La necesidad por encontrar nuevas fuentes de madera con calidad para tonelería, tiene el propósito de preservar las actuales zonas forestales, además de disminuir los costos, al no utilizar barriles de roble extranjeros empleando roble colombiano durante la maduración de estas bebidas. En Colombia, el roble se encuentra ubicado en departamentos como Nariño, Boyacá, Huila, Santanderes, Antioquia, Caldas Cauca, Caquetá, Cundinamarca y Tolima. Su temperatura óptima de crecimiento va de 16 
a $25^{\circ} \mathrm{C}$ y se encuentra ubicado entre los 1500 a 2400 m sobre el nivel del mar (Morea, 1997; Mosedale et al., 1999). El objetivo del presente trabajo fue estudiar la composición química en cuanto a sustancias marcadoras de envejecimiento del roble colombiano sin tostar y tostado a $180{ }^{\circ} \mathrm{C}$ por tres horas que según Monedero et al. (1998) son las mejores condiciones para obtener una buena composición fenólica; simulando el proceso de quemado en la elaboración de los barriles, y observar la posibilidad de realizar estudios posteriores al roble colombiano para evaluar su posible utilización en la fabricación de barriles destinados a la maduración de las bebidas destiladas.

\section{MATERIALES Y MÉTODOS}

\section{Método Analítico}

Extracción de compuestos fenólicos a partir del roble colombiano. A dos muestras de aserrín de $1 \mathrm{~g}$ de roble colombiano (Quercus humboldtii), tomado dos $\mathrm{Km}$ al norte de Pamplonita (Norte de Santander), una tostada $\left(180{ }^{\circ} \mathrm{C}\right.$ por tres horas) y la otra sin tostar, se les adicionó por separado una mezcla de $100 \mathrm{ml}$ metanol:agua en una proporción 1:1. Esta preparación se dejo actuar durante 24 horas a temperatura ambiente, y finalmente se procedió a realizar la extracción conforme al método descrito por Fernández de Simón et al. (1996).

\section{Determinación y cuantificación de siringaldehído y vainillina}

Análisis cromatográfico. Las muestras se analizaron por HPLC en fase reversa, en un cromatógrafo líquido Agilent $1100^{\circledR}$ con bomba cuaternaria, columna supelcosil LC18.DB de $5 \mu \mathrm{m} 25 \mathrm{~cm} \times 4.5 \mathrm{~mm}$ a $40{ }^{\circ} \mathrm{C}$. Para la elusión de los compuestos se empleó una fase móvil compuesta por dos solventes: Fase móvil A compuesta por agua/ácido acético glacial (98/2) y una fase móvil B compuesta por metanol/agua/ácido acético glacial (70/28/2) (Batista de Aquino et al., 2006) en gradiente con un flujo del solvente de $1.25 \mathrm{ml}$ minuto $^{-1}$. Se cuantificó a una longitud de onda de $300 \mathrm{~nm}$. Las inyecciones cromatográficas se llevaron a cabo por triplicado para cada una de las muestras, previa filtración a través de filtros millipore de $0.45 \mu \mathrm{m}$.

Cuantificación siringaldehído. Con el fin de determinar los intervalos en los que el método analítico presenta proporcionalidad lineal entre la concentración de siringaldehído y el área de pico encontrado (Curva de calibración), se utilizó un sistema modelo constituído por concentraciones crecientes del patrón de siringaldehído marca FLUKA $(>97 \%)$, con inyecciones cromatográficas por triplicado de $20 \mu \mathrm{l}$. Este compuesto fue identificado mediante comparación de los tiempos de retención en combinación con su espectro UV-Vis, con aquellos obtenidos de los estándares, teniendo un límite de detección de 130 partes por billón (ppb). Tomando como base un estándar de siringaldehído, se elaboró la recta de calibración a partir de siete soluciones patrón entre 0.150 y $25.20 \mathrm{mg} \mathrm{L}^{-1}$ usando como solvente etanol grado HPLC al 40\%. Para verificar la reproducibilidad del sistema cromatográfico, las inyecciones cromatográficas se llevaron a cabo por triplicado para cada uno de los patrones (Figura 1).

Cuantificación de vainillina. La curva de calibración se hizo utilizando un patrón de Vainillina marca FLUKA (>98\%), con inyección por triplicado de $20 \mu \mathrm{l}$. A partir de un estándar de vainillina, se elaboró la recta de calibración a partir de siete soluciones patrón entre 0.10 y $25.20 \mathrm{mg} \mathrm{L}^{-1}$ usando como solvente etanol grado HPLC. Para verificar la reproducibilidad del sistema cromatográfico, las inyecciones 


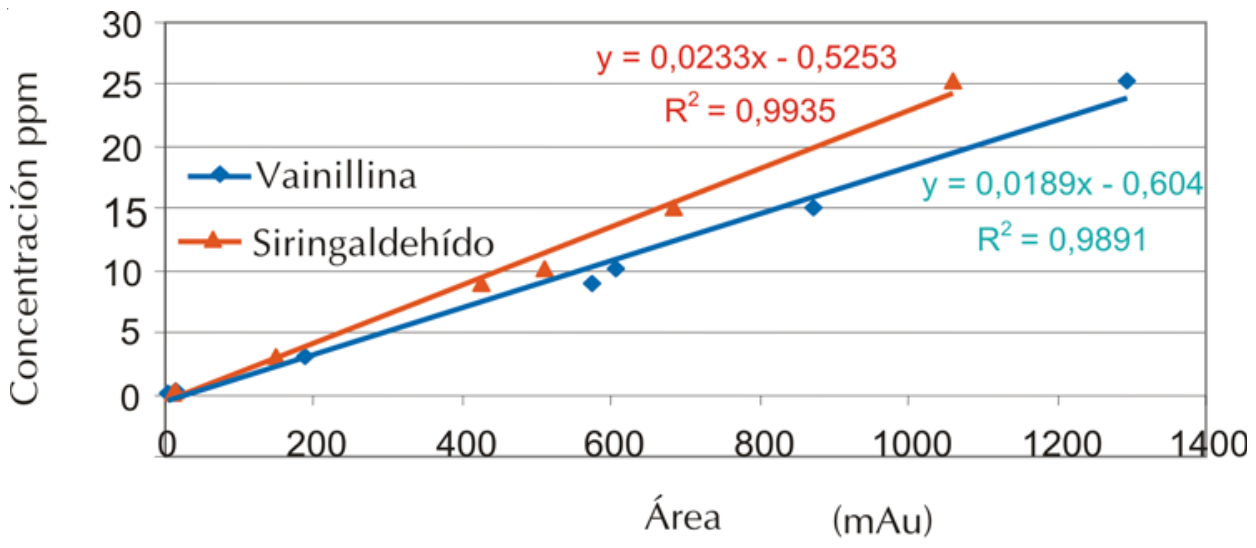

Figura 1. Rectas de calibración para soluciones hidroalcohólicas al $40^{\circ} \mathrm{GL}$ de siringaldehído y vainillina

cromatográficas se llevaron a cabo por triplicado para cada uno de los patrones. Como se puede apreciar en la figura 1, el modelo de regresión lineal simple para la vainillina es: $y$ $=0,0189 x-0,604$, teniendo en cuenta el intervalo de confianza de la pendiente $(0.016$ - 0.021) con un nivel de confianza del 95\%. Este compuesto fue identificado mediante comparación de sus tiempos de retención en combinación con el espectro UV-Vis con aquellos obtenidos de los estándares, con un límite de detección de 70 ppb.

Tratamiento de la información. Finalmente los datos obtenidos se analizaron mediante el paquete estadístico SPSS (v.13.0) utilizando ANOVA para observar diferencias significativas $(\operatorname{Pr}<0.05)$ entre las medias de siringaldehido y vainillina del roble colombiano con los encontrados en la literatura (Cadahia et al., 2001).

\section{RESULTADOS Y ANÁLISIS}

Extracción de compuestos fenólicos en roble colombiano tostado a $180{ }^{\circ} \mathrm{C}$

Los extractos de Quercus humboldtii obtenidos revelaron la presencia de siringaldehído y vainillina en muestras de roble tostadas a $180{ }^{\circ} \mathrm{C}$ durante tres horas. Además, se presentaron otros compuestos, los cuales no eran motivo de investigación en este trabajo (Figura 2). Estos compuestos también han sido descritos en robles de diferentes especies con y sin tratamiento

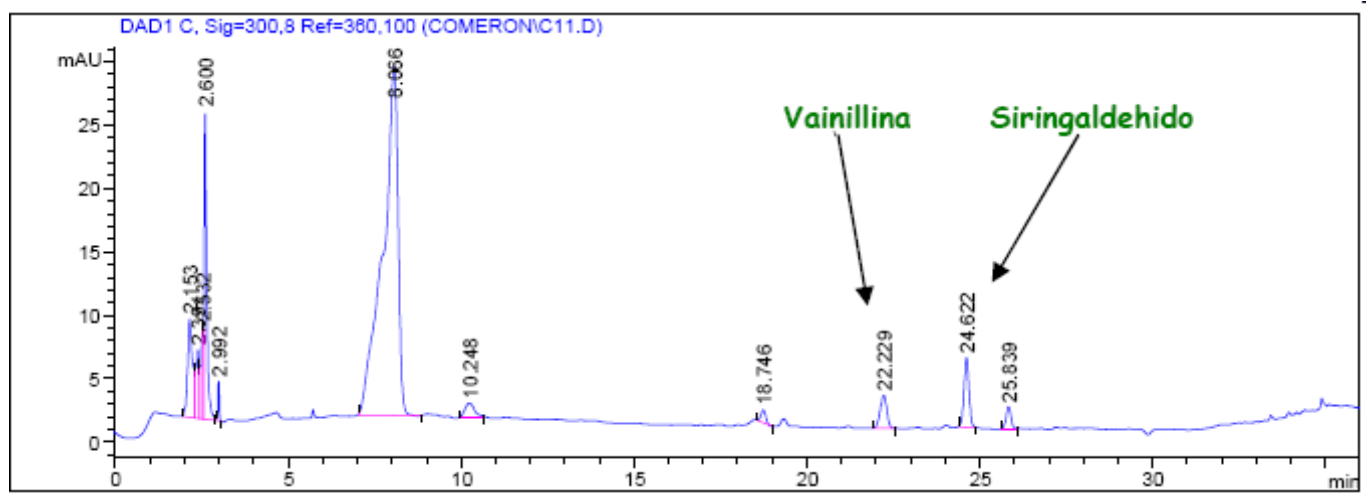

Figura 2. Cromatograma de Quercus humboldtii tostado a $180^{\circ} \mathrm{C}$ por tres horas 
térmico por varios autores aún cuando no presentan las concentraciones respectivas de cada compuesto (Chatonnet et al., 1989; Dubois, 1989; Artajona, 1991; Clímaco y Borralho, 1996; Pérez et al., 1999).

En la figura $3 \mathrm{~A}$ se puede apreciar la variabilidad existente entre las concentraciones de siringaldehído y vainillina del roble colombiano con respecto a otros robles Españoles (Quercus robur, $Q$. petraea, Q. pyrenaica) sometidos a tostado de intensidad medio $\left(160-170{ }^{\circ} \mathrm{C}\right.$ durante 35 minutos aproximadamente), roble Francés $(Q$. robur, $Q$. petraea) y roble Americano (Quercus alba) (Cadahia et al., 2001). Después del ANOVA aplicado a las distintas concentraciones de vainillina en las maderas de roble tostadas, se estableció que existen diferencias significativas $(\operatorname{Pr}<0.05)$ entre el roble colombiano (Quercus Humboldtii) y los demás robles (Español, Americano y Francés); también se presentaron diferencias estadísticas significativas entre las especies del roble Español (Q. robur, Q. petraea, Q. pyrenaica, $Q$. faginea), Francés (Q. robur, $Q$. petraea) y Americano (Q. alba). Estas diferencias pueden ser por causa de los tratamientos térmicos, ya que el tiempo de exposición al calor fue mayor para el roble colombiano (tres horas); mientras que para el resto de robles la exposición fue menor. Estas diferencias también se pueden ocasionar por las distintas especies de roble y el origen geográfico de los mismos (Pérez et al., 1999; Fernández et al., 2003).

Quercus Humboldtii únicamente presenta similitud en la concentración de vainillina con el roble Español Quercus faginea; siendo para este de $64.6 \mu \mathrm{g} \mathrm{g}^{-1}$ y para el roble colombiano de $64 \mu \mathrm{g} \mathrm{g}^{-1}$. En cuanto a la concentración de siringaldehído, Quercus humboldtii no presenta similitud con ninguno de los robles con los cuales fue comparado, ya que su concentración es de $92 \mu \mathrm{g} \mathrm{g}^{-1}$, la cual es inferior al resto de robles, mientras que Quercus alba (roble Americano) presenta la mayor cantidad de siringaldehído $\left(536 \mu \mathrm{g} \mathrm{g}^{-1}\right)$ superando en un amplio margen al roble colombiano. La relación de siringaldehido/vainillina para el roble colombiano se fija en 1.43 atendiendo a las concentraciones encontradas, concordando con el trabajo de Puech y Jouret (1982), que tras analizar las concentraciones de siringaldehído y vainillina en muestras de origen perfectamente conocido, Ilegaron a la conclusión que la relación siringaldehído/ vainillina debe estar comprendida entre 1.4 y 2.5, lo cual indicaría que existe un equilibrio entre los productos de degradación de la lignina (siringaldehído y vainillina).

\section{Extracción de compuestos fenólicos en roble colombiano sin tostar.}

En los análisis realizados por cromatografía líquida a los extractos del roble colombiano sin tratamiento térmico no se encontraron los aldehídos fenólicos (vainillina y siringaldehído), contrastando con los resultados reportados por Fernández de Simón et al. (1996) y Cadahia et al. (2001) en donde encontraron pequeñas cantidades de estos compuestos en roble Español sin tostar (Figura 3B). El ANOVA teniendo en cuenta las concentraciones de siringaldehído y vainillina para todos los robles, permitió observar que el roble colombiano presenta diferencias estadísticas significativas $(P r>$ 0.05) con todos los robles con los que se comparó, esto puede ser debido al secado ya que en esta fase se originan compuestos fenólicos (Chatonnet et al., 1994; Fernández de Simón et al., 1999). Considerando los datos obtenidos del roble colombiano, con y sin tratamiento térmico, se puede señalar que las concentraciones de sustancias marcadoras de envejecimiento incrementan significativamente debido al tratamiento térmico que sufre la madera; concordando 
A

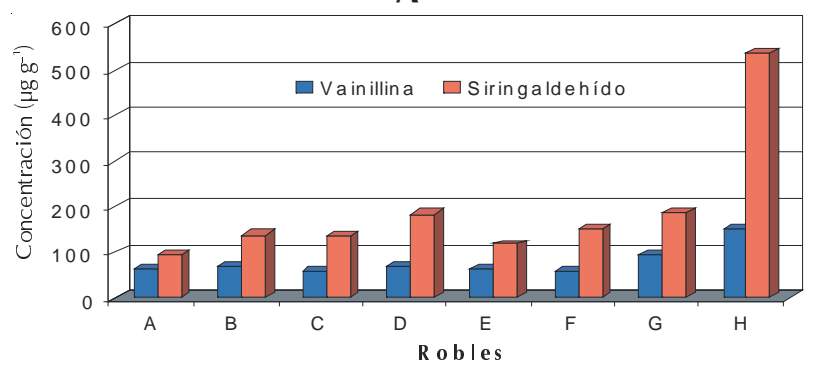

B

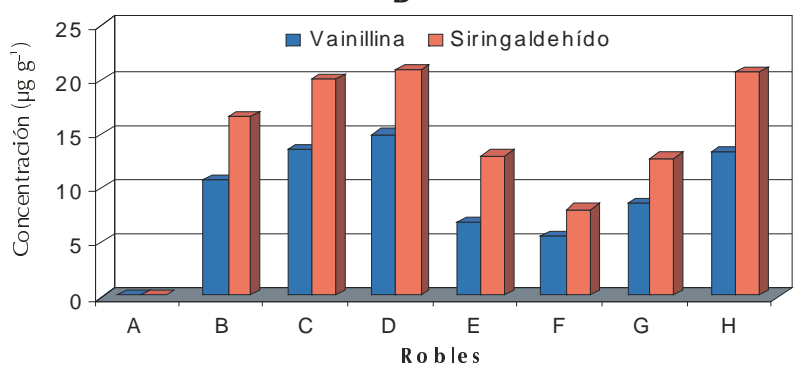

Figura 3. Concentración de SME entre robles Colombiano (A: Q. humboldtii), Español (B: Q. robur, C: Q. petraea, D: Q. pyrenaica y E: Q. faginea), Francés (F: Q. robar y G: Q. petraea) y Americano $(\mathrm{H}:$ Q alba) con tratamiento térmico $(\mathbf{A})$ y sin con tratamiento térmico (B).

con trabajos anteriores sobre roble Francés y Americano (Chatonnet et al., 1994; Swan et al., 1993). La explicación a este incremento es probablemente debido a la termodegradación de la lignina por medio de procesos de depolimerización y posterior degradación hidrolítica oxidativa (Monties, 1992; Vivas et al., 1996).

\section{CONCLUSIONES}

- Las concentraciones de siringaldehído en el roble tostado fue de $92 \mu \mathrm{g} \mathrm{g}^{-1}$ y de 64 $\mu \mathrm{g} \mathrm{g}{ }^{-1}$ para la vainillina fijando la relación siringaldehido/vainillina en 1.43, lo que indica un equilibrio entre estos aldehídos.

- Quercus Humboldtii únicamente presenta similitud en la concentración de vainillina con el roble Español (Quercus faginea) sometido a tratamiento térmico.

- El proceso de tostado influye claramente en la composición del roble colombiano, ya que no se detectó la presencia de siringaldehido y vainillina en el roble sin tostar. Pudiendo ser útil este parámetro para diferenciarlo de robles Españoles y Americanos.

- Se recomienda estudiar más las temperaturas de tostado, ya que estas influencian la composición del roble y para establecer un mejor control de estas sustancias que finalmente influyen en la calidad de las bebidas alcohólicas maduradas en esta matriz.

\section{BIBLIOGRAFÍA}

Ancín, C.; Garde, T.; Torrea, D. y Jiménez N. 2004. Extraction of volatile compounds in model wine from different oak woods. Effect of $\mathrm{SO}_{2}$. Food Research International $37(4): 375-383$
Artajona, S. 1991. Caracterización del roble según su origen y grado de tostado, mediante la utilización de CG y HPLC. Viticultura y Enología Profesional 14(5/6): 61-72 
Batista de Aquino, F.; Rodríguez, S., Ferreira, N. y Soares, R. 2006. Simultaneous determination of aging markers in sugar cane spirits. Food Chemistry 98 (3):569-574

Bozhinov, A. y Bekalov, N. 1983. Oak extract in the aging of wine distillates. Lozar. Vinar 32(2):21-24

Cadahia, E.; Muñoz, L.; De simón, B. y Garcia, C. 2001. Changes in low molecular weight phenolic compounds in Spanish, French, and American oak woods during natural seasoning and toasting. Journal of Agricultural and Food Chemistry 49(4):1790-1798

Clímaco, M. y Borralho, A. 1996. Influences de technologies d'èlevage dans les transformations des composants de I'arôme des vins rouges. In $A$. Loonvaud-funel (Ed), Oenologie 95-5e Symposium international d'oenologie Lavoisier, Paris, p415-418

Chatonnet, P.; Boidron, J. y Pons, M. 1989. Incidence du traitement thermique du bois de chene sur la composition chimique. $2^{a}$ partie. Èvolution de certains composés en function de I'intensité de brulage. Connaissance Vigne Vin 23(4):223-250

Chatonnet, P.; Boidron, J.; Dubordieu, D. y Pon,s M. 1994. Évolution de certains composés volatils du bois de chêne au cours de son séchage. Premires resultants. Journal International des Sciences de la Vigne et du vin 28(4):359-380

Chatonnet, P. y Dubourdieu, D. 1998.Comparative study of the characteristics of American white oak (Quercus alba) and European oak (Quercus petraea and Q. robur) for production of barrels used in barrel aging of wines. American Journal of Enology and Viticulture 49(1):79-85

Delgado, T.; Gómez, C. y Villarroya, B. 1990. Relationships between phenolics compounds of low molecular weight as indicators of aging conditions and quality of brandies. American Journal of Enology and Viticulture $41(4): 342-345$

Dubois, P. 1989. Apport du fût de chène neuf a l'arôme des vins. Revista Francesa de Oenologia 29(120):19-24

Fernández de Simón, B.; Conde, E.; Cadahía, E. y García, M. 1996. Low-molecularweight phenolic compounds in woods of Spanish, French and American oak. Journal des Sciences et Techniques de La Tonnellerie 2:13-23

Fernández de Simón, B.; Cadahia, E.; Conde, E. y Garcia, M. 1999. Ellagitatannins in woods of Spanish, French and American oaks. Holzforschung 53(47):147-150

Fernández de Simón, B.; Cadahía, E. y Galocha, J. 2003. Volatile Compounds in a Spanish red wine Aged in barrels made of Spanish, French, and American oak word. Journal of Agricultural and Food Chemistry 51(26):7671-7678

Garde, T. y Ancín, C. 2006. Effect of oak barrel type on the volatile composition of wine. Storage time optimization. Food Science and Technology 39 (3):199-205 
Jaganathan, J. y Dugar, J. 1999. Authentication of straight whiskey by determination of the ratio of furfural to 5-hydroxymethyl-2furaldehyde. Journal of AOAC International 82(24):997-1001

Lo Coco, F.; Valentín, C.; Novelli, V. y Ceccon, L. 1995. Liquid chromatograph determination of 2furaldehyde and 5- hydroxymethyl2 -furaldehyde in beer. Analytica Chimica Acta 306(57):57-64

Mangas, J.; Rodríguez, R. y Moreno, J. 1996. Evolution of aromatic and furanic congeners in the maturation of cider brandy: A contribution to its characterization. Journal of Agricultural and Food Chemistry 44(12):3303-3307

Morea, L. 1997. Sinexi S.A. Robledales en Colombia. URL:http//www.google. com.co.html [Accedido: 07-042006]

Monedero, L.; Olalla, M.; Quesada, J.; Villalón, M.; López, H. y López, M. 1998. Exhaustion techniques in the selection and description of phenolic compounds in jerez wine extracts obtained by an accelerated aging technique. Journal of Agricultural and Food Chemistry 46(5):1754-1764

Monties, B. 1992. Chemical composition oakwood: phenolic compound and their relation with physical and chemical properties related to quality of wines and spirits. (The wood and the quality of wines and spirits). International Journal of Vine and Wine Sciences, special issue 59-74
Mosedale, J. y Savill, P. 1996. Variation of heartwood phenolics and oak lactones between the species and phonological types of Quercus petraea and $Q$. robur. Forestry $69(1): 47-54$

Pérez, M.; Sanz, J. y Cabezudo, M. 1999. Determination of volatile compounds in hydroalcoholic extracts of French and American oak wood. American Journal of Enology and Viticulture 50(2):162-165

Puech, J. y Jouret, C. 1982. Dosage des aldéydes aromatiques des eaux-devie conservées en futs de chene:Detection D'adulteration. Annales des Falsifications, de L'Expertise Chimique et Toxicologique 75(85):81-90

Quesada, J.; Villalón, M.; López, H. y López, M. 1996. Influence of aging factors on the furanics aldehide contens of matured brandies: aging markers. Journal of Agricultural and Food Chemistry 44(6):1378-1381

Swan, J.; Reid, K.; Howie, D. y Howlet, S. 1993. Study of the effects of air and kiln drying of cooperage oakwood. En: Cantagrel, R. (Ed). Elaboration et Connaissance des Spiritueux. Lavoisier, Paris, p557-561

Vivas, N.; Glories, Y. y Doneche, B. 1996. Thought on natural dryingof oakwood for barrels production. Revue Franc Fenol 4:348-52 